\title{
ON COMPLETENESS OF QUASI-PSEUDOMETRIC SPACES
}

\author{
SEITHUTI P. MOSHOKOA
}

Received 4 July 2004 and in revised form 21 April 2005

We discuss completeness in terms of a notion of absolute closure. This will be done in the context of separated quasi-pseudometric spaces and bitopological spaces. The notion is equivalent to the classical notion of completeness when restricted to the class of metric spaces.

\section{Introduction and preliminaries}

The notion of completeness in metric spaces and that of completing a metric space are traditionally discussed in terms of Cauchy sequences. The main reason being that this concept deals precisely with the issue of convergence of sequences in the sense that every convergent sequence is a Cauchy sequence. The paper deals with completion in a setting that avoids explicit reference to Cauchy sequences.

First we recall some definitions which will be used in the paper, see also $[3,5,9]$. Let $X$ be a set and let $d: X \times X \rightarrow[0, \infty)$ be a function such that for all $x, y, z \in X$,

(i) $d(x, x)=0$,

(ii) $d(x, y) \leq d(x, z)+d(z, y)$.

Then $d$ is called a quasi-pseudometric. If $d$ is a quasi-pseudometric on $X$, then its conjugate denoted by $d^{-1}$ on $X$ is such that $d^{-1}(x, y)=d(y, x)$ for all $x, y \in X$. Certainly $d^{-1}$ is a quasi-pseudometric. Let $d^{*}=d \vee d^{-1}$. Then $d^{*}$ is also quasi-pseudometric on $X$. If a quasi-pseudometric $d$ on $X$ satisfies $d(x, y)=d(y, x)$ for all $x, y \in X$ in addition to (i) and (ii), then $d$ is called pseudometric. A pseudometric $d$ that satisfies $d(x, y)=0$ if and only if $x=y$ is called a metric.

Now if $d$ is a quasi-pseudometric such that $d(x, y)+d(y, x)>0$ for all $x \neq y$, then $d$ is said to separate points in $X$. The set $X$ equipped with a quasi-pseudometric $d$ is said to be bounded if its diameter $\delta(X)=\sup \{d(x, y): x, y \in X\}$ exists. The open ball with centre $x \in X$ and radius $r>0$ is $B(x, r)=\{y \in X: d(x, y)<r\}$, and by $\tau(d)$ we denote the topology on $X$ induced by $d$. We will say that a sequence $\left\{x_{n}\right\}$ in $(X, d)$ converges to $x \in X$ if $\lim _{n} d\left(x, x_{n}\right)=0$. When $d$ is a separating pseudometric, then it is a metric, that is, the topology $\tau(d)$ is Hausdorff.

By analogy with the topological case, in which a $T_{2}$ space is said to be absolutely closed (H-closed) when it is closed in every $T_{2}$ space that contains it, we recall the following. 
Definition 1.1 (see [6]). A metric space $(X, d)$ is absolutely closed if its image is a closed subset of any space in which it can be embedded.

Theorem 1.2 (see [6]). The metric space $(X, d)$ is complete if and only if $X$ is absolutely closed.

Since the paper discusses completeness in spaces more general than metric spaces we provide a generalization of Definition 1.1 by considering a space $(X, d)$, where $d$ can be one of the functions we defined above, namely, a metric, a pseudometric, or a quasipseudometric on $X$. By $M$ we will denote a class of spaces. In fact, we denote by $M_{m}$ the class of all metric spaces, by $\mathcal{M}_{p}$ the class of all pseudometric spaces, and by $\mathcal{M}_{q}$ the class of all quasi-pseudometric spaces. The class $\mathcal{M}$ to be considered in each context will be explicitly mentioned.

Firstly, we provide the following.

Definition 1.3. The space $(X, d)$ is absolutely closed in $\mathcal{M}$ if $(X, d)$ is closed in every space $(\bar{X}, \bar{d})$ that contains it, that is, $(\bar{X}, \bar{d}) \in \mathcal{M}$ and $\left.\bar{d}\right|_{X \times X}=d$. When this is the case we will simply say that $(X, d)$ is $M$-absolutely closed.

So, Theorem 1.2 becomes as follows.

Theorem 1.4. Let $(X, d)$ be a metric space. Then $(X, d)$ is complete if and only if $(X, d)$ is $M_{m}$-absolutely closed.

The paper [4] discusses a topological problem of characterizing the type of absolute "closure" properties possessed by compact spaces. In the paper, we discuss an analogous problem and completeness for the pseudometric spaces and quasi-pseudometric spaces by constructing explicit extensions for these spaces.

\section{Completeness without Cauchy sequences}

The problem of completing a space is one of the classical extension problems in analysis and topology. Intuitively, a complete metric space is one in which every sequence that "should" converge does converge, in other words it is a space which cannot be extended as far as convergence of its sequences is concerned.

We introduce the following definition and show that it provides us with an equivalent notion of the classical Cauchy-sequence completeness in the cases of a metric space and pseudometric space.

Definition 2.1. Let $(X, d)$ be a space. Then $(X, d)$ is said to be $M$-convergence complete if for every space $(\bar{X}, \bar{d}) \in \mathcal{M}$ such that $(X, d) \subseteq(\bar{X}, \bar{d})$, a sequence $\left\{x_{n}\right\}$ in $X$ that converges to a point $\bar{x}$ in $\bar{X}$ also converges to some point $x$ in $X$.

Then the following result is immediate.

Proposition 2.2. Let $(X, d)$ be a metric space. The following conditions are equivalent:

(i) $X$ is complete,

(ii) $X$ is $M_{m}$-convergence complete,

(iii) $X$ is $M_{m}$-absolutely closed. 
Proof. (i) $\Leftrightarrow$ (iii) follows by Theorem 1.4. Next we see that (ii) $\Leftrightarrow(\mathrm{i})$ is clear, because in a metric space $(X, d)$ a convergent sequence is Cauchy.

We give a simple example.

Example 2.3. Complete pseudometric spaces are $M_{p}$-convergence complete spaces, because in a pseudometric space a convergent sequence is a Cauchy sequence and a Cauchy sequence in a complete pseudometric space has a limit.

Now we give an analogue of Proposition 2.2 in the context of pseudometric spaces. These spaces are not Hausdorff and we have the following.

Proposition 2.4. Let $(X, d)$ be a pseudometric space. Then the following conditions are equivalent:

(i) $X$ is complete,

(ii) $X$ is $M_{p}$-convergence complete.

Observe that Proposition 2.2 does not hold for quasi-pseudometric spaces. These spaces are not symmetric and therefore there are several inequivalent notions of a Cauchy sequence which lead to different notions of a complete space, see $[7,8]$ for example. In the next section we will examine the analogue of Proposition 2.2 in nonsymmetric spaces. In particular we discuss $M$-convergence completeness in quasi-pseudometric spaces.

\section{Completeness of quasi-pseudometric spaces}

We start this section by further discussing extensions of spaces, and generalize our results to extensions of bounded quasi-pseudometric spaces.

We first consider the following.

Proposition 3.1. Let $(X, d)$ be a pseudometric space. If $(X, d)$ is $M_{q}$-convergence complete, then $(X, d)$ is complete.

Proof. We consider an arbitrary Cauchy sequence $\left\{x_{n}\right\}$ in $X$. If $\left\{x_{n}\right\}$ converges in $(\bar{X}, \bar{d}) \in$ $M_{q}$ that contains $(X, d)$, then it also converges in $X$, since $X$ is $M_{q}$-convergence complete, that is, every Cauchy sequence in $X$ converges in $X$. Hence $(X, d)$ is complete.

When $(X, d)$ in Proposition 3.1 is a metric space, we get the following.

Corollary 3.2. Let $(X, d)$ be a metric space. If $(X, d)$ is $M_{q}$-convergence complete, then $(X, d)$ is complete.

The following example shows that $\mathbb{R}$ with the usual metric is not $M_{q}$-convergence complete.

Example 3.3. Let $X=\mathbb{R}$ with the usual metric. The sequence $\left\{(-1)^{n}\right\}$ in $X$ is not convergent in $X$. Let $\bar{X}=\mathbb{R} \cup\{*\}$. Define $\bar{d}: \bar{X} \times \bar{X} \rightarrow[0, \infty)$ by $\bar{d}(x, y)=|x-y|$, if $x, y \in \mathbb{R}$, $\bar{d}(*, *)=0$,

$$
\bar{d}(x, *)=\left\{\begin{array}{ll}
-x+1 & \text { if } x<-1, \\
2 & \text { if }-1 \leq x \leq 1, \\
x+1 & \text { if } x>1,
\end{array} \quad \bar{d}(*, x)= \begin{cases}-1-x & \text { if } x<-1, \\
0 & \text { if }-1 \leq x \leq 1, \\
x-1 & \text { if } x>1 .\end{cases}\right.
$$


Then $(\bar{X}, \bar{d})$ is a quasi-pseudometric space that contains $(X, d)$. Now $\bar{d}\left(*, x_{n}\right)=0$ for all $n \in \mathbb{N}$, hence $\bar{d}\left(*, x_{n}\right) \rightarrow 0$ as $n \rightarrow \infty$, where $x_{n}=(-1)^{n}$. It follows that a sequence $\left\{x_{n}\right\}$ converges in $\bar{X}$ but not in $X$. Therefore $\mathbb{R}$ is not $\mathcal{M}_{q}$-convergence complete. Since $X$ is complete when equipped with the usual metric, we see that the converse of Proposition 3.1 does not hold.

Generally, we show that if a bounded quasi-pseudometric space $(X, d)$ is closed in every quasi-pseudometric space $(\bar{X}, \bar{d})$ that properly contains $(X, d)$, then $X$ is an empty set: in strong contrast with the metric space case.

Theorem 3.4. Let $(X, d)$ be a quasi-pseudometric space such that $d$ is bounded and separates points in $X$. Then $(X, d)$ is $M_{q}$-absolutely closed if and only if $X$ is the empty set.

Proof. Suppose that $X$ is an empty set. Then every bounded quasi-pseudometric space $(\bar{X}, \bar{d})$ contains an empty set as a closed subset, hence we are done. Conversely, suppose that $X$ is not an empty set. We construct a quasi-pseudometric space that contains $X$ in the following way: for a point $b \notin X$, let $\bar{X}=\{b\} \cup X$. Define $\bar{d}: \bar{X} \times \bar{X} \rightarrow[0, \infty)$ by

$$
\bar{d}(x, y)= \begin{cases}d(x, y) & \text { if } x, y \in X \\ 0 & \text { if } x=b \text { and } y \in \bar{X} \\ M & \text { if } x \in X \text { and } y=b\end{cases}
$$

where $M=\delta(X)$. Now $\bar{d}(x, x)=0$ for all $x \in \bar{X}$ trivially holds, also the inequality $\bar{d}(u, v) \leq$ $\bar{d}(u, w)+\bar{d}(w, v)$ certainly holds for every $u, v, w \in \bar{X}$. Hence $\bar{d}$ is a quasi-pseudometric and $X \subset \bar{X}$. Now since $X$ is nonempty, let $a \in X$. For any $r>0$, we have $d(b, a)=0<$ $r$. Thus every open ball about $b$ has a nonempty intersection with $X$, in particular $a \in$ $B(b, r) \cap X$. This shows that $X$ is not closed in $(\bar{X}, \bar{d})$.

We now give the following.

Theorem 3.5. Let $(X, d)$ be a bounded quasi-pseudometric space such that $d$ separates points. Then the following properties are equivalent:

(i) $X$ is $M_{q}$-convergence complete,

(ii) every sequence in $X$ is $\tau(d)$-convergent.

Proof. (ii) $\Rightarrow$ (i). If every sequence $\left\{x_{n}\right\}$ in $X$ converges, then we are done. Assume (i). Let $\left\{x_{n}\right\}$ be a sequence in $X$. Define $\bar{X}=X \cup\{b\}$, where the point $b$ does not belong to $X$. Next we define a function $\bar{d}: \bar{X} \times \bar{X} \rightarrow[0, \infty)$ by

$$
\bar{d}(x, y)= \begin{cases}d(x, y) & \text { if } x, y \in X, \\ 0 & \text { if } x=b \text { and } y \in \bar{X}, \\ M & \text { if } x \in X \text { and } y=b,\end{cases}
$$

where $M=\delta(X)$. Then we have $X \subset \bar{X}$ and $\bar{d}$ is a quasi-pseudometric on $\bar{X}$. Also $\lim _{n} \bar{d}(b$, $\left.x_{n}\right)=0$ for every sequence $\left\{x_{n}\right\}$ in $X$. Hence the sequence $\left\{x_{n}\right\}$ converges in $\bar{X}$ and it therefore converges in $X$. This shows that (i) $\Rightarrow$ (ii). 
When $(X, d)$ is a bounded metric space, we get the following.

Corollary 3.6. Let $(X, d)$ be a bounded metric space. Then for the following properties:

(i) $X$ is $M_{q}$-convergence complete,

(ii) every sequence in $X$ is $\tau(d)$-convergent,

(iii) $X$ is complete,

(i) $\Leftrightarrow($ ii), (i) $\Rightarrow($ iii), and (ii) $\Rightarrow$ (iii).

Proof. (i) $\Leftrightarrow$ (ii) follows by Theorem 3.5. (i) $\Rightarrow$ (iii) and (ii) $\Rightarrow$ (iii) are clear.

Let us observe that (iii) does not imply (i) and that (iii) does not imply (ii) by Example 3.3, hence in Corollary 3.6, the statements (i), (ii), and (iii) are not equivalent.

We now give an example.

Example 3.7. Consider a set $X$ which consists of one point, say $X=\{a\}$, and the set $\bar{X}$ that consists of two points, say $\bar{X}=\{a, b\}$. We define a function $d: X \times X \rightarrow[0, \infty)$ by $d(x, y)=$ 0 for all $x, y \in X$ (observe that $x=y=a$, in this case) and a function $\bar{d}: \bar{X} \times \bar{X} \rightarrow[0, \infty)$ defined by

$$
\bar{d}(x, y)= \begin{cases}d(x, y) & \text { if } x, y \in X, \\ 0 & \text { if } x=b \text { and } y \in \bar{X} \\ 1 & \text { if } x \in X \text { and } y=b\end{cases}
$$

Then $d$ and $\bar{d}$ are, respectively, quasi-pseudometrics on $X$ and $\bar{X}$, we also have $X \subset \bar{X}$. Furthermore the smallest neighborhood of the point $a$ is $\{a\}$ while the smallest neighborhood of the point $b$ is $\bar{X}$. In particular, the assumptions of Theorem 3.5 holds.

When trying to establish the $M$-convergence completeness for quasi-pseudometric spaces, it was necessary to consider an "internal" description of sequences in $X$ that converge to some point outside $X$. We now characterize those bounded quasi-pseudometric spaces which admit extensions in terms of real-valued uniformly continuous maps.

For the next two results, namely, Theorems 3.8 and 3.10, we consider $\mathbb{R}$ with the quasipseudometric $u$ on $\mathbb{R}$ defined by $u(x, y)=(y-x) \vee 0$, for all $x, y \in \mathbb{R}$, and its conjugate $l=u^{-1}$ on $\mathbb{R}$. Note that $\tau(u)$ is the upper topology on $\mathbb{R}$ and $\tau(l)$ is the lower topology in $\mathbb{R}$.

Theorem 3.8. Let $(X, d)$ be a bounded quasi-pseudometric space such that $d$ separates points. Then the following statements are equivalent:

(i) $(X, d)$ admits a quasi-pseudometric space extension $(\bar{X}, \bar{d})$ such that $X \subseteq \bar{X}$ and $\left.\bar{d}\right|_{X \times X}=d$,

(ii) there exists a pair $f$ and $g$ of real-valued uniformly continuous maps on $X$ satisfying, for $x, y \in X$,

(a) $u(f(x), f(y)) \leq d(x, y)$,

(b) $l(g(x), g(y)) \leq d(x, y)$,

(c) $d(x, y) \leq f(x)+g(y)$. 
Proof. We will show that (i) $\Rightarrow$ (ii). Suppose (i) holds. Without loss of generality we will assume that $\bar{X}=X \cup\{*\}$, where the point $*$ does not belong to $X$. Then the functions $f:(X, d) \rightarrow \mathbb{R}$, where $\mathbb{R}$ is equipped with $\tau(u)$, and $g:(X, d) \rightarrow \mathbb{R}$, with $\mathbb{R}$ equipped with $\tau(l)$, defined by $f(x)=\bar{d}(*, x)$ and $g(x)=\bar{d}(x, *)$, respectively, are uniformly continuous and satisfy (a), (b), and (c). Hence (i) $\Rightarrow$ (ii). Conversely suppose (ii) holds. Fix $f$ and $g$. Then for a point $*$ that does not belong to $X$ we extend $d$ to $\bar{X}=X \cup\{*\}$, such that the extension is also a quasi-pseudometric on $\bar{X}$ in the following way: let $\bar{d}(x, *)=g(x)$ and $\bar{d}(*, x)=f(x)$. Next, we put $\bar{d}(*, *)=0$. Then $\bar{d}$ is a quasi-pseudometric on $\bar{X}$ and $X \subset \bar{X}$. It follows that $(\bar{X}, \bar{d})$ contains $(X, d)$, and (i) holds. Hence the proof is complete.

By an extension pair $(f, g)$ of $(X, d)$ on the extension $(\bar{X}, \bar{d})$ we will refer to functions $f$ and $g$ as in Theorem 3.8.

We now introduce the following definition.

Definition 3.9. Let $(X, d)$ be a quasi-pseudometric space. A sequence $\left\{x_{n}\right\}$ in $X$ is called an $f$ null sequence if there exists an extension pair $(f, g)$ such that $\left\{f\left(x_{n}\right)\right\} \rightarrow 0$ as $n \rightarrow \infty$.

From our characterization on extension of bounded quasi-pseudometric spaces we get the following.

Theorem 3.10. Let $(X, d)$ be a bounded quasi-pseudometric space such that $d$ separates points. Then the following statements are equivalent:

(i) $X$ is $M_{q}$-convergence complete,

(ii) every sequence in $X$ is $\tau(d)$-convergent,

(iii) there exists an extension pair $(f, g)$ such that every sequence $\left\{x_{n}\right\}$ in $X$ is an $f$ null sequence.

Proof. (i) $\Rightarrow$ (iii). Suppose (i) holds. Let $(\bar{X}, \bar{d})$ be an extension of $(X, d)$. By Theorem 3.8 there exists an extension pair $(f, g)$. We will show that every sequence $\left\{x_{n}\right\}$ is an $f$ null sequence, where $f$ is as defined in the proof of Theorem 3.8. Let $\left\{x_{n}\right\}$ be an arbitrary sequence in $X$ that converges to a point in $\bar{X}$. Without loss of generality we may assume that $\left\{x_{n}\right\}$ converges to $* \notin X$ and $\bar{X}=\{*\} \cup X$ as in Theorem 3.8, then we get $f\left(x_{n}\right) \stackrel{n \rightarrow \infty}{\longrightarrow} 0$. Hence $\left\{x_{n}\right\}$ is an $f$ null sequence. Thus (iii) holds. The implications (i) $\Leftrightarrow$ (ii) follow by Theorem 3.5. Next we show that (iii) $\Rightarrow$ (ii). Suppose (iii) holds. Fix an extension pair $(f, g)$. Now if every sequence $\left\{x_{n}\right\}$ in $X$ is an $f$ null sequence, then every sequence $\left\{x_{n}\right\}$ is $\tau(d)$-convergent by (uniform) continuity of $f$ on $X$. Then (ii) holds. Finally the implication (iii) $\Rightarrow(\mathrm{i})$ is clear.

Remark 3.11. Theorem 3.5 is a special case of Theorem 3.10. To see this we consider an extension $\bar{X}=X \cup\{b\}$ of $X$ where the point $b$ does not belong to $X$ as in the proof of the theorem. Let $M=\delta(X)$. Then $d$ admits an extension denoted by $\bar{d}$ as in Theorem 3.5 to $\bar{X}$. Clearly we obtain an extension pair $(f, g)$ on $X$ defined for all $x \in X$, by $f(x)=\bar{d}(b, x)$ and $g(x)=\bar{d}(x, b)$, respectively. Then every sequence $\left\{x_{n}\right\}$ in $X$ is an $f$ null sequence.

Our next example shows that when $(X, d)$ is unbounded, then statements (i) and (ii) in Theorem 3.5 and in Corollary 3.6 may not be equivalent, that is, we have (ii) $\Rightarrow$ (i) but not conversely. 
Example 3.12. Consider $X=\mathbb{N} \times\{0\}$ with a function $d: X \times X \rightarrow[0, \infty)$ defined by $d(a$, $b)=|x-y|$ for all $a=(x, 0), b=(y, 0) \in X$. Then $(X, d)$ is an unbounded (quasi-pseudo) metric space. Let $\bar{X}=X \cup X$, where $X=\mathbb{N} \times\{1\}$. So for each $a \in X$ there is a point $a \in X^{\prime}$ above $a$, where $a=(x, 1)$, and $x \in \mathbb{N}$ (we refer the reader to the double arrow space, in $[1$, page 414$])$. We write $a=(x, 1)$ and $\dot{b}=(y, 1)$ for $\dot{a} \neq \hat{b}$, in $\dot{X}$, and the corresponding points in $X$ are $a=(x, 0)$ and $b=(y, 0)$ with $x, y \in \mathbb{N}$. Now, define a function $\bar{d}: \bar{X} \times \bar{X} \rightarrow$ $[0, \infty)$ by $\bar{d}(a, a)=1$ and

$$
|x-y|=\left\{\begin{array}{l}
\bar{d}(\dot{a}, \hat{b}) \\
\bar{d}(\dot{a}, b) \\
\bar{d}(a, b) \\
\bar{d}(a, b)
\end{array} \quad \text { if } a \neq b .\right.
$$

Then $\bar{d}$ is a quasi-pseudometric on $\bar{X}$. Clearly (ii) $\Rightarrow(\mathrm{i})$, that is, a sequence $\left\{x_{n}\right\}$ in $X$ that converges to a point say $a ́ \in \bar{X}$ also converges to some $a \in X$. But not every sequence in $X$ is convergent. For instance, the sequence $\left\{x_{n}=(n, 0): n \in \mathbb{N}\right\}$ in $X$ is not $\tau(d)$ convergent. Therefore in Theorem 3.5, the implication (ii) $\Rightarrow$ (i) does not hold.

Remark 3.13. In general, we see that Theorem 3.5 holds for a finite quasi-pseudometric space $(X, d)$ since every such space has a maximal element with respect to $\leq$ defined by $x \leq y$ if and only if $y \in \mathrm{cl}_{\tau(d)}\{x\}$, where $x$ and $y$ belong to $X$. More generally, consider a quasi-pseudometric space $(X, d)$ with a maximal element $x_{\infty}$, that is, $x \leq x_{\infty}$ for all $x \in X$. Now the condition $x \leq x_{\infty}$ is equivalent to $x_{\infty} \in \mathrm{cl}_{\tau(d)}\{x\}$ which is equivalent to the property that $V$ is a neighborhood of $x_{\infty}$ if and only if $V=X$. For instance in Example 3.7 the maximal element $x_{\infty}$ in $(\bar{X}, \bar{d})$ is $b$. Furthermore the neighborhood of the maximal element $x_{\infty}$ is $\bar{X}$. In this example, every sequence converges.

A close examination of Example 3.7 and Remark 3.13 prompts us to ask this question: must every quasi-pseudometric space to which Theorem 3.5 applies have a maximal element?

The following result provides an affirmative answer to our question in a special case.

Theorem 3.14. Let $(X, d)$ be a compact quasi-pseudometric space such that every sequence in $X$ converges, then there exists a maximal element, and every sequence converges to that element.

Proof. Let $(X, d)$ be compact. Define $x^{\dagger}=\{y \in X: x \leq y\}$, where $x \leq y$ if $y \in \operatorname{cl}_{\tau(d)}\{x\}$. First we will verify that $x^{\dagger}$ is closed in $X$ when $X$ is equipped with the topology induced by $d$. Suppose that $z \notin x^{\dagger}$, then $x \leq z$ is false, that is $z \notin \mathrm{cl}_{\tau(d)}\{x\}$. Now $X-\mathrm{cl}_{\tau(d)}\{x\}$ is open and contains $z$. So let $V=X-\mathrm{cl}_{\tau(d)}\{x\}$. Then $V \cap x^{\dagger}=\varnothing$. To see this we let $t \in V \cap x^{\dagger}$. Then $t \in V$ and $t \in \mathrm{cl}_{\tau(d)}\{x\}$ as $x \leq t$. So we get $t \in V-\mathrm{cl}_{\tau(d)}\{x\}$ which is impossible. This shows that $x^{\dagger}$ is closed. Next let $\mathscr{F}$ be the following set $\left\{x^{\dagger}: x \in X\right\}$. We will show that $\mathscr{F}$ has a finite intersection property and this will complete our proof, since, because of compactness of $(X, \tau(d))$ we will then have $\cap x^{\dagger} \neq \varnothing$, and $z$ such that $z \in \cap x^{\dagger}$ is clearly a maximal element of $(X, \leq)$. Let $x, y \in X$. We will show that $x^{\dagger} \cap y^{\dagger} \neq \varnothing$. Consider the 
following sequence: $x, y, x, y, x, y, \ldots$ in $X$, that is, $x_{n}=x$ when $n \in \mathbb{N}$ is odd and $x_{n}=y$ when $n \in \mathbb{N}$ is even. Since every sequence in $X$ converges, then $\left\{x_{n}\right\}$ converges, say, to $z$. It remains to show that $z \in x^{\dagger} \cap y^{\dagger}$. Suppose that $z \notin x^{\dagger} \cap y^{\dagger}$, then we either have

(i) $z \notin x^{\dagger}$ and $z \in y^{\dagger}$, or

(ii) $z \in x^{\dagger}$ and $z \notin y^{\dagger}$.

If (i) holds, then $z \notin x^{\dagger}$ so $x \leq z$ is false, thus $z \notin \mathrm{cl}_{\tau(d)}\{x\}$. Clearly $V=X-\mathrm{cl}_{\tau(d)}\{x\}$ is an open neighborhood of $z$ and hence the sequence $\left\{x_{n}\right\}$ is eventually in $V$. This gives $x \in V$ which is a contradiction as $x \in \operatorname{cl}_{\tau(d)}\{x\}$, therefore $z \in x^{\dagger}$. Similarly if (ii) holds, we have $z \in y^{\dagger}$. Thus in both cases we have $z \in x^{\dagger} \cap y^{\dagger}$. So $\mathscr{F}$ has the finite intersection property, as required.

The following example shows that the conclusion of Theorem 3.5 holds in (noncompact) quasi-pseudometric spaces without a maximal element.

Example 3.15. Let $X$ be the first uncountable ordinal with its usual well-ordering. Then $x \in X$ if and only if $\{y \in X: y \leq x\}$ is countable. $X$ does not have a maximal element. Define a function $d: X \times X \rightarrow[0, \infty)$ by

$$
d(x, y)= \begin{cases}0 & \text { if } y \leq x \\ 1 & \text { if } y>x\end{cases}
$$

Then $d$ is a quasi-pseudometric on $X$. Also, $B(x, 1 / 2)=x \downarrow$ is countable, consequently, $\tau(d)$ is the topology on $X$ with the basic open sets of the form $x^{\downarrow}$. Consider any sequence $\left\{x_{n}\right\}$ in $X$. Clearly the sets $x_{n}^{\downarrow}$ are countable and bounded for each $n \in \mathbb{N}$, and so is $\cup x_{n}^{\downarrow}$. So every sequence in $X$ converges with respect to the topology $\tau(d)$, and $X$ is not compact. In fact $X$ is sequentially compact but not compact, see $[2,5]$ for example.

\section{Closed subspaces of $M$-convergence complete spaces}

In this section, we discuss closed subspaces of $M$-convergence complete spaces. Since in a complete metric space $(X, d)$ if $A$ is a closed subspace of $X$, then $A$ is complete, then an $M$-convergence complete $(M$-absolutely closed) analogue of this result is: in an $M$ convergence complete metric space $(X, d)$ a closed subspace $A$ of $X$ is $\left(M_{m}\right.$-absolutely closed) $M_{m}$-convergence complete. Also, it is well-known that a complete subspace of an arbitrary metric space is closed, phrased in our context, as an $M_{m}$-convergence complete subspace $A$ of a metric space $(X, d)$ is closed.

In the context of quasi-pseudometric spaces we have the following result concerning subspaces of $M_{q}$-convergence complete spaces.

We need a lemma.

Lemma 4.1. Let $(X, d) \in M_{q}$ and let $A$ be a subspace of $X$. If $(\dot{A}, \dot{d}) \in M_{q}$ contains $A$, then there exists $(\bar{X}, \bar{d}) \in M_{q}$ such that $(\bar{X}, \bar{d})$ contains $(X, \bar{d})$.

Proof. Suppose that $A$ is a subspace of $(X, d) \in \mathcal{M}_{q}$ and $(A, d) \in M_{q}$ contains $A$. Without loss of generality, we may assume that $A=A \cup\{*\}$ and $* \notin X$. Now, let $\bar{X}=X \cup\{*\}$. 
Define a function $\bar{d}: \bar{X} \times \bar{X} \rightarrow[0, \infty)$ by

$$
\bar{d}(x, y)= \begin{cases}d(x, y) & \text { if } x, y \in X, \\ 0 & \text { if } x=y=*, \\ \inf _{a \in A}\{\dot{d}(*, a)+d(a, y)\} & \text { if } x=*, y \in X, \\ \inf _{a \in A}\{d(x, a)+d(a, *)\} & \text { if } x \in X, y=* .\end{cases}
$$

Easily we have $\bar{d}(x, x)=0$ for all $x \in \bar{X}$. To show that $\bar{d}(u, v) \leq \bar{d}(u, w)+\bar{d}(w, v)$ holds for all $u, v, w \in \bar{X}$ it will be enough if we can show that $d(u, v) \leq \bar{d}(u, *)+\bar{d}(*, v)$ for all $u, v \in X$ and $* \in \bar{X}$. Now $d(u, v) \leq d(u, a)+d(a, v)$ holds for all $u, v \in X$ and all $a \in A$. Since $\dot{d}(a, *) \geq 0$ and $\dot{d}(*, a) \geq 0$ in $A$, then we have

$$
d(u, v)<d(u, a)+\dot{d}(a, *)+\dot{d}(*, a)+d(a, v)
$$

for all $u, v \in X$ and all $a \in A$. In particular, the following holds:

$$
d(u, v) \leq \inf _{a \in A}\{d(u, a)+\dot{d}(a, *)+\dot{d}(*, a)+d(a, v)\} .
$$

Hence for all $u, v \in X$, we have

$$
d(u, v) \leq \bar{d}(u, *)+\bar{d}(*, v) .
$$

Then it follows that $\bar{d}$ is a quasi-pseudometric space on $\bar{X}$ that extends both $\bar{d}$ and $d$, also $\bar{X}$ contains both $A$ and $X$.

Theorem 4.2. Let $(X, d)$ be an $M_{q}$-convergence complete quasi-pseudometric space such that $d$ separates points in $X$. Suppose $A \subseteq X$ is a closed subspace, then $\left(A, d_{A}\right)$ is $M_{q^{-}}$ convergence complete.

Proof. Let $(X, d)$ be $M_{q}$-convergence complete quasi pseudometric space such that $d$ separates points in $X$ and $A \subseteq X$ be a closed subspace. Suppose a sequence $\left\{a_{n}\right\}$ in $A$ converges to some point $*$ in $(\hat{A}, \dot{d}) \in \mathcal{M}_{q}$ that contains $A$. Without loss of generality we assume that $A=\{*\} \cup A$. By Lemma 4.1 we obtain $(\bar{X}, \bar{d})$ that contains $X$. Then $\left\{a_{n}\right\}$ converges to $*$ in $\bar{X}$ with respect to $\bar{d}$. Now since $\left\{a_{n}\right\}$ belongs to $X$ and $X$ is $M_{q}$-convergence complete, it follows that $\left\{a_{n}\right\}$ converges to some point $x \in X$. This implies that $x \in A$ as $A$ is closed in $X$. Hence $\left(A, d_{A}\right)$ is $M_{q}$-convergence complete.

We can easily construct an example to shows that the converse of Theorem 4.2 does not hold, that is, a subspace $A$ of an $M_{q}$-convergence complete quasi-pseudometric space $(X, d)$ can be $M_{q}$-convergence complete without being closed, in contrast to the metric space case. 


\section{Completeness in bitopological spaces}

Definition 5.1. Let $(X, d)$ be a quasi-pseudometric space and $d^{*}=d \vee d^{-1}$. Then a sequence $\left\{x_{n}\right\}$ in $\left(X, d^{*}\right)$ converges to a point $x$ if it converges with respect to $\tau\left(d^{*}\right)$.

Remark 5.2. We observe that in the space $\left(X, d^{*}\right)$ the sequence $\left\{x_{n}\right\}$ converges to a point $x$ if and only if it converges to $x$ with respect to $\tau(d)$ and $\tau\left(d^{-1}\right)$. We also observe that if both $d$ and $d^{-1}$ are separating, then $d^{*}$ is a metric on $X$.

Definition 5.3 (see [9]). We say that the space $\left(X, d^{*}\right)$ is complete if every $d^{*}$-Cauchy sequence converges in $X$ with respect to $\tau\left(d^{*}\right)$.

Finally we give the following result whose proof follows from Proposition 2.2, Definitions 5.1 and 5.3, and Remark 5.2. It complements Proposition 2.2.

Theorem 5.4. Let $(X, d)$ be a quasi-pseudometric space such that $d$ and $d^{-1}$ separate points. Then the following conditions are equivalent:

(i) $\left(X, d^{*}\right)$ is complete,

(ii) $\left(X, d^{*}\right)$ is $M_{m}$-convergence complete,

(iii) $\left(X, d^{*}\right)$ is $M_{m}$-absolutely closed.

In conclusion, we see that a notion of completeness is equivalent to $\mu_{p}$-convergence completeness. Furthermore, completeness in metric spaces is equivalent to $M_{m}$-absolute closure. In contrast with the metric space case we observe that a nonempty quasipseudometric space may be $M_{q}$-convergence complete without being $M_{q}$-absolutely closed. Finally, we see that in the case of bitopological spaces equivalent notions of completeness as in the context of metric spaces are obtained.

\section{Acknowledgments}

This work has been supported by the National Research Foundation under Grant number 2053847. The author also wishes to thank Professor Sergio Salbany for his kindness, valuable suggestions, and comments which were crucial for the completion of this paper. Finally, the reports from anonymous referees which improved the quality of the paper are greatly acknowledged.

\section{References}

[1] R. Engelking, General Topology, 2nd ed., Sigma Series in Pure Mathematics, vol. 6, Heldermann, Berlin, 1989.

[2] J. Ferrer and V. Gregori, A sequentially compact noncompact quasipseudometric space, Monatsh. Math. 96 (1983), no. 4, 269-270.

[3] P. Fletcher and W. F. Lindgren, Quasi-Uniform Spaces, Lecture Notes in Pure and Applied Mathematics, vol. 77, Marcel Dekker, New York, 1982.

[4] D. Harris, Extension closed and cluster closed subspaces, Canad. J. Math. 24 (1972), 1132-1136.

[5] H.-P. A. Künzi, A note on sequentially compact quasipseudometric spaces, Monatsh. Math. 95 (1983), no. 3, 219-220.

[6] W. J. Pervin, Foundations of General Topology, Academic Press Textbooks in Mathematics, Academic Press, New York, 1964. 
[7] I. L. Reilly, P. V. Subrahmanyam, and M. K. Vamanamurthy, Cauchy sequences in quasipseudometric spaces, Monatsh. Math. 93 (1982), no. 2, 127-140.

[8] S. Romaguera and S. Salbany, On the Niemytzki-Tychonoff theorem for distance spaces, Fasc. Math. (1990), no. 19, 223-231.

[9] S. Salbany, Bitopological Spaces, Compactifications and Completions, Mathematical Monographs of the University of Cape Town, no. 1, Department of Mathematics, University of Cape Town, Cape Town, 1974.

Seithuti P. Moshokoa: Department of Mathematics, Applied Mathematics and Astronomy, University of South Africa, P.O. Box 392, Pretoria 0003, South Africa

E-mail address: moshosp@unisa.ac.za 


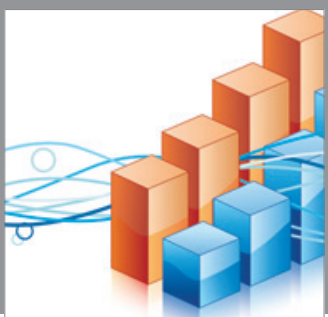

Advances in

Operations Research

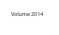

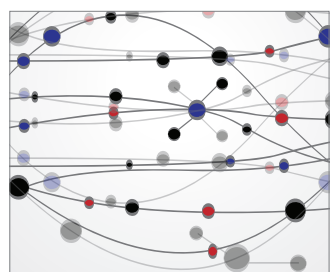

\section{The Scientific} World Journal
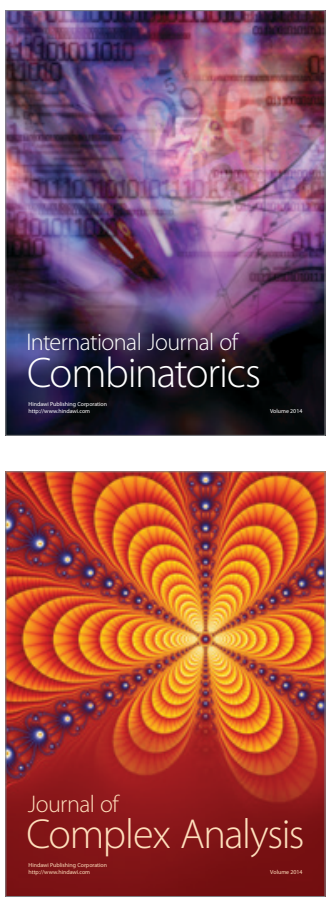

International Journal of

Mathematics and

Mathematical

Sciences
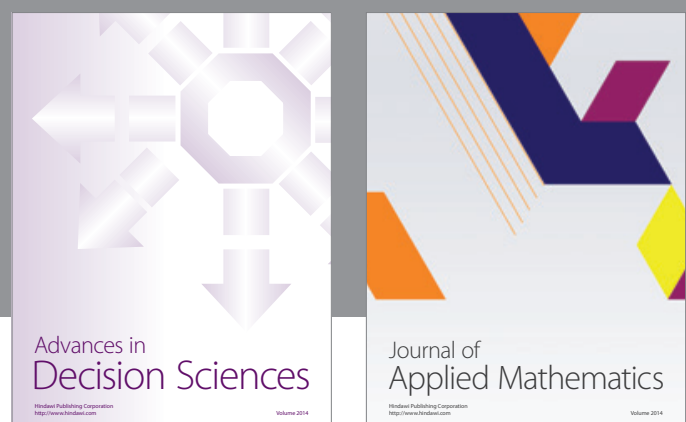

Journal of

Applied Mathematics
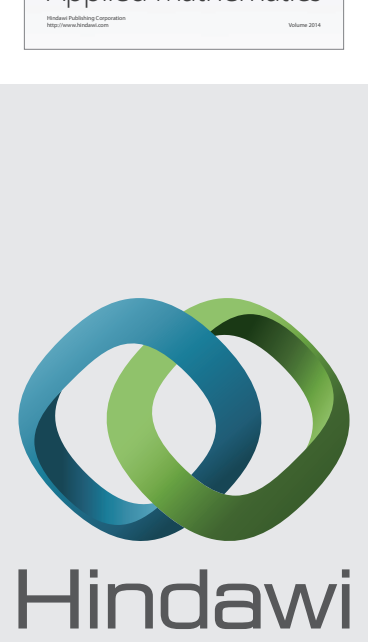

Submit your manuscripts at http://www.hindawi.com
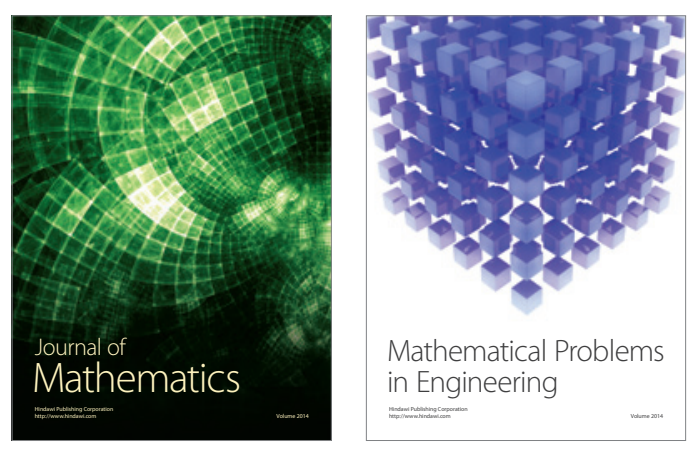

Mathematical Problems in Engineering
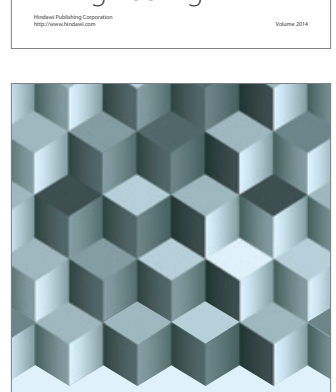

Journal of

Function Spaces
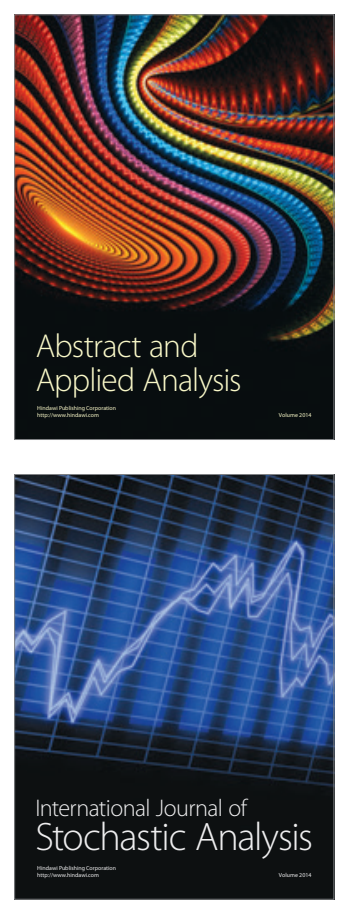

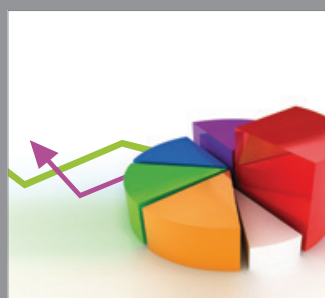

ournal of

Probability and Statistics

Promensencen
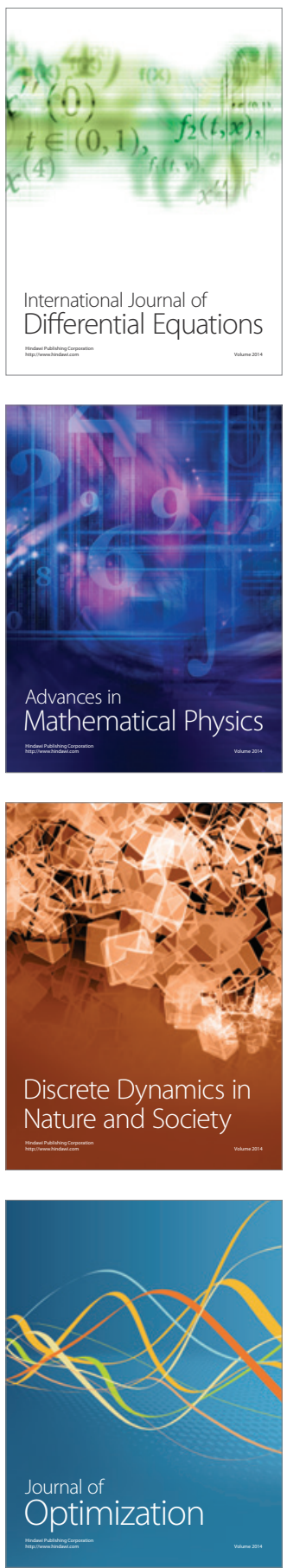